\title{
Gamification in Late-Life Depression: A Serious Game for a Serious Problem
}

\author{
Vincenza Frisardi* and Roberto Caragnulo \\ Geriatric and Neurorehabilitation Department, Institute of Scientific Research IRCCS Arcispedale Santa Maria Nuova, Italy \\ *Corresponding author: Vincenza Frisardi, Geriatric and Neurorehabilitation Department, Institute of Scientific \\ Research IRCCS Arcispedale Santa Maria Nuova, Italy
}

\begin{tabular}{|c|c|}
\hline ARTICLE INFO & ABSTRACT \\
\hline Received: 慧 February 26, 2020 & Citation: Vincenza F, Roberto C. Gamification in Late-Life Depression: A Serious Game for a \\
\hline Published: March 03, 2020 & Serious Problem. Biomed J Sci \& Tech Res 26(2)-2020. BJSTR. MS.ID.004320. \\
\hline
\end{tabular}

\section{Mini Review}

In the older population, one of the serious health concerns associated with negative outcomes such as comorbidity, loss of physical autonomy, excessive use of health care resources, and increased mortality is the onset of mood disorder [1]. Late-life depression (LLD) is an umbrella term to define depressed mood in people older than 65 years. It can have a variety of presentations: as recurrent disease stemming from earlier life (early onset depression [EOD]); as new-onset depression (late-onset depression [LOD]); as a mood disorder secondary to a general medical condition; or as mood symptoms secondary to substance or medication use [2]. Mistakenly, LLD is believed as a normal part of aging. For older people, depression could be the epiphenomenon of underlying biological processes (vascular changes, metabolic disturbances and neurodegenerative diseases); conversely, it could be the leading cause of cognitive impairment [3]. LLD is challenging to distinguish it from dementia, as both these diagnoses have such overlapping phenotypic profiles, especially when depression affects motivation, attention and focus abilities [3]. Additionally, attributing depression to old age may be a barrier to treatment seeking, and there are many potential reasons for poor treatment adherence in older adults, including stigma, negative patient beliefs about treatment, physical and psychiatric comorbidity, and costs of care [3].

Reports indicate that $15-27 \%$ of older adults in the community and up to $37 \%$ in primary care settings experience depressive symptoms [4]. Among the US population it was estimated that between 1.2 and 1.8 million adults 65 years of age and older had a depressive disorder. Rates of depressive disorders in community-

dwelling elderly persons over the age of 65 vary by race/ethnicity, with the highest rates of depressive disorders being among the Hispanic/Latino populations. Among individuals in this age group who are in nursing homes, the prevalence of depression is significantly higher $(49.6 \%)[5,6]$. With the aging population, this estimate has expected to increase exponentially. Although LLD is associated with a substantial individual and societal burden, it receives much less attention than many other geriatric medical disorders. To minimize the burden of depression for older adults and their loved ones, it requires global efforts. In fact, depression hits caregivers of affected people too with long-term negative health effects and increasing the mortality risk among them. Summarizing, LLD modify the own health trajectories leading to poorer outcomes in subjects with other chronic diseases, affecting caregiver family and social network functioning too [7].

The Diagnostic and Statistical Manual of Mental Disorders (DSM5) defines major depressive disorder by the presence of depressed mood or a striking loss of interest or pleasure in activities along with 5 or more of the following associated symptoms: changes in appetite or weight, sleep, energy, concentration, and psychomotor activity, feelings of inappropriate guilt or worthlessness and recurrent thoughts of death or suicide [8]. All of these symptoms could open the road to other significant geriatric syndromes as malnutrition, sarcopenia, physical deconditioning with the wellknown serious consequences. Worldwide, LLD is underdiagnosed and inadequately treated with serious impact on the whole health system. Strategies for an early LLD detection or to counteract it 
are challenging. Although several evidence-based psychological (behavioral cognitive therapy, cognitive bibliotherapy, problem solving therapy, brief psychodynamic therapy, life review therapy) and pharmacological treatments are available, even in high-income countries less than half of patients receive treatment and this occurs mainly in the older people [9]. Clinicians used to treat LLD by focusing on antidepressants and this could represent a suboptimal care for old people especially in an advisable perspective of "deprescribing". Consequently, it is mandatory to invest the full scale of knowledge to increase the possibilities of treatment and care especially if our goal is to reduce the disease burden. We could be able to perform our goal firstly by facilitating an early detection of subclinical depression. Subsequently, through a specific evaluation of the therapeutic effects of any treatments in order to establish a priori what kind of results we are able to reach in a personalized way. Furthermore, we must support interventions that look beyond the depressed individual and include caregiver, professionals and other members of that individual's social network.One of the ways in which that can be done is through the technological application that move away from "conventional" internet approach, towards gamification and artificial intelligence.

Gamification is a relatively new trend that focuses on applying games to non-game contexts and electronic technology has effectively contributed to the rise of gamification in many industries. While many fields have taken advantage of the potential of gamification as a construct to develop business, social and training applications (see for example Business, Marketing and e-Learning), the digital healthcare domain has started to exploit this emerging trend recently [10,11]. Both personalized health care and computer games place the individual in the center of the action, and this is the goal of any patient-centered medical practice. With the advent of mobile technologies, such as smartphones or digital wristwatch and their apps, the process of being reminded has become more automated. Applying gamification to daily routine reminder tasks could motivate people to participate and engage in setting goals and objectives for themselves. Currently, some experience of gamification in health derived from chronic disease rehabilitation whereas physical activity and mental focus are the principal area of interest in wellness, but evidence are still limited [12]. The deployment and design of serious games for psychotherapy has recently been studied in young people [13]. Unfortunately, no sufficient data are available in adults and old people. Nonetheless, the amount of senior who are confident with technological device or social network is increasing. In the UK, approximately $89 \%$ of adults use the Internet, and users aged 65 to 74 years increased from $52 \%$ to $78 \%$ in 2011 . Approximately $42 \%$ of people of all genders aged $\geq 75$ years are now Internet users $[14,15]$. Therefore, policy makers and stakeholders might intercept this "abilities" and promoting them in order to increase the opportunities in the care and treatment of this disorder. The use of video games in psychotherapy could be a tactical way to approach to several difficulties physical and psychological only if therapists gain familiarity with gaming equipment. As before mentioned, to fight LLD-related negative outcomes, the involvement of health professionals, caregiver and community in a safety network for depressed patients is extremely important. Very often, the cultural obstacle to implementation in the daily practice of technological devices even comes from the health professionals who are unfamiliar with new technological approach and that require longer learning curves than patients do. Several concerns could arise about the effectiveness of this modality compared to faceto-face approaches. However, a large body of evidence supported the equal efficacy of Internet intervention compared to face-to-face intervention in the treatment of depression. Whether it is using games to serve a serious purpose or gamify a serious purpose, the goal is to help individuals reduce mental health complaints or improve their mental wellbeing. However, how do serious games perform on improving mental health? Are there evidence-based serious games for the treatment or prevention of mental health symptoms? Several issues emerge normally when the field is unexplored yet.

As above-mentioned, the major difficulty for internet-based technological intervention is to have this knowledge applied in routine care especially when we face with several cultural and administrative barriers. For example, health professionals could think that technological interventions will take over their jobs, or they are unaware about the potential resources of new devices or technological applications. Caregivers and patients could fear to become dependent on Internet and to develop gaming disorder or simply to feel skeptical. Finally, administrative integration of these digital interventions in mosthealth care system couldbecomplicated. Despite these concerns, recent evidence has demonstrated that game-based interventions could improve the cognition (both fluid and crystallized cognitive functions) in healthy older adults as well in patients with cognitive impairment. The positive effects could result from specific action on cerebral circuits (intrinsic and extrinsic motivation) and/or by the influence of physical movement on neuronal biological processes (enhancements in white matter integrity in older adults and myelin sheet regeneration, increased important neurotrophic factor levels). Several interventional studies have indicated a positive impact of specific exercises on depressive symptoms reduction. In fact, exercise-induced changes are linked to neuronal protection by maximizing cardiorespiratory fitness [16], increasing brain-derived neurotrophic factor [17] and reducing cortisol level [18].

Sometime depressed people don't accept conventional therapy because they are unaware about the problem by declining any type of intervention (individual or group therapy). Sometimes we assist also to the high rate of treatment dropout. In this regard, serious game make intervention more acceptable for patients and so easy to apply. Moreover, the format of game represents in these subjects a suitable trigger for the meso-cortico-limbic circuitry, one of the 
principal neuronal substrates of pleasure stimulating motivation. Recently, Stojan and Voelcker-Rehage presented a comprehensive overview of the efficiency and underlying neurophysiological processes of "exergaming training" (a neologism that relies on technology that tracks body movement or reaction) in older adults. Besides the heterogeneity of findings in this new field, studies that compared exergaming to traditional types of exercise, showed similar or slightly superior effects of exergaming on executive functions but not on other cognitive domains. This might be an indicator that exergaming is a promising approach to preserve and facilitate cognitive and brain health in healthy older adults. The authors concluded that exergaming might be a useful alternative to traditional exercise to motivate older people to habitually engage in physical activity [19]. However, further researches are needed to determine influencing factors that contribute to intervention efficacy, to discover underlying mechanisms and to apply tailored intervention. This is very important especially in the era of artificial intelligence and machine learning. Gamification applied to LLD could be a modern way to alleviate mood or behavioral disorders by increasing interactions with friends, family, caregivers or other patients in a virtual world. Social isolation is one of dramatic feature of LLD and therefore home internet-based approach could compensate the loneliness feeling occurring variably. We should take advantage from this phenomenon to apply serious game with therapeutic approach. To reach the full potential of gamification, it is necessary to build e-Health solutions on well-founded theories that exploit the core experience and psychological effects of game mechanics. For this purpose, it is mandatory to promote several projects in this field. Contextualizing gamification in the research field of late-life mood disorders, we could seize its usefulness in different way: protective and preventing, therapeutic, for assessment, educational and informatics category. Thus, the application of serious games might be considered as a protective factor, a potential non-pharmacological strategy but also as tools to educate health professionals and finally to collect big data.

In order to prevent LLD, exergaming could be used in people who do not want to go out for a walking or to the gym because their physical impairment (for example, people affected by arthrosis or after an orthopedic surgery). The positive impact of games in this domain suggests that game-based interventions might contribute to support old people and their caregivers to face with initial aging-related problems before they convert in pathology. If we think to the possibilities to connect people with the same medical problems or social situation, serious games may create a virtual world where people "transfer" themselves and reinforce their individual know-how to face with their psychological problem. Furthermore, sharing personal problems with other people but protecting the own intimate world of emotions through a virtual reality, could represent a way to win the fear to be judged and the social stigma too. Moreover, gamification could be useful in the targeting subject who are not appealed from the game-based approach, in fact, listlessness, apathy, demotivation, absence of curiosity represent some phenotypic characteristic of an early mood impairment. Furthermore, it could be useful to monitor the medication adherence to therapy, to enter in the virtual community of players and to share disease-related uneasiness. There is a worldwide shortage of health workers, and this issue requires innovative education solutions. Serious gaming and gamification education have the potential to provide a quality, cost-effective, novel approach that is flexible, portable, and enjoyable and allow interaction. If therapist gain confidence in the use of digital devices for depression treatment, they will be more satisfied and motivated to cope with geriatric patients affected by depression. Gamebased digital applications for depression therapy is a rather new research area. Consequently, policy makers and stakeholders need to intercept this "abilities" in favor to step up in the knowledge of this field. We need to face several issues about where and how the gamification can be applied and how it fits in the current systems for health care and how implement it with artificial intelligence. However, there should be no doubt that international consortium and projects are welcomed in order to increase the possibilities to intervene in LLD and change this field completely.

\section{Acknowledgment}

None.

\section{Conflict of Interest}

We have no disclosure to make that qualifies as a conflict of interest.

\section{References}

1. Krishnan KR, Delong M, Kraemer H, Carney R, Spiegel D, et al. (2002) Comorbidity of depression with other medical diseases in the elderly. Biol Psychiatry 52(6): 559-588.

2. Aziz R, Steffens DC (2013) What are the causes of late-life depression? Psychiatr Clin North Am 36(4): 497-516.

3. Frisardi V, Panza F, Farooqui AA (2011) Late-life depression and Alzheimer's disease: The glutamatergic system inside of this mirror relationship. Brain Res Rev 67(1-2): 344-355.

4. Hybels CF, Pieper CF (2009) Epidemiology and Geriatric Psychiatry. American Journal of Geriatric Psychiatry 17(8): 627-663.

5. Gonzalez O, McKnight-Eily LR, Strine T, et al. (2010) Current depression among adults-United States, 2006 and 2008. MMWR Morb Mortal Wkly Rep 59: 1229-1235.

6. (2012) IOM (Institute of Medicine). The mental health and substance abuse workforce for older adults: In whose hands? The National Academies Press, Washington, DC, USA.

7. Pinquart M, Sorensen S (2003) Differences between caregivers and noncaregivers in psychological health and physical health: A meta-analysis. Psychology and Aging J 18(2): 250-267.

8. (2013) American Psychiatric Association, Task F. Diagnostic and statistical manual of mental disorders DSM-5. ( $5^{\text {th }}$ Edn.).

9. Olfson M, Pincus HA (1996) Outpatient mental health care in nonhospital settings: distribution of patients across provider groups. American Journal of Psychiatry 153: 1353-1356.

10. Christians, Gerald (2018) The Origins and Future of Gamification (2018). Senior Theses: 254 
11. Brezinka V (2008) Treasure Hunt-a serious game to support psychotherapeutic treatment of children. Stud Health Technol Inform 136: 71-76

12. Sardi L, Idri A, Fernández Alemán JL (2017) A systematic review of gamification in e-Health.

J Biomed Inform 71: 31-48.

13. Christie GI, Shepherd M, Merry SN, Hopkins S, Knightly S, et al. (2019) Gamifying CBT to deliver emotional health treatment to young people on smartphones. Internet Interv 18: 100286.

14. (2016) Prescott C. Internet users in the UK - Office for National Statistics.

15. (2016) Of com. Internet use and attitudes bulletin. 2016.

16. Stubbs B, Rosenbaum S, Vancampfort D, Ward PB, Schuch FB (2016) Exercise improves cardiorespiratory fitness in people with depression:

ISSN: 2574-1241

DOI: $10.26717 /$ BJSTR.2020.26.004320

Vincenza Frisardi. Biomed J Sci \& Tech Res

(C) This work is licensed under Creative

Submission Link: https://biomedres.us/submit-manuscript.php
A meta-analysis of randomized control trials. J Affect Disord 190: 249253.

17. Laske C, Banschbach S, Stransky E, Bosch S, Straten G, et al. (2010) Exercise-induced normalization of decreased BDNF serum concentration in elderly women with remitted major depression. Int J Neuropsychopharmacol 13: 595-602.

18. Beserra AHN, Kameda P, Deslandes AC, Schuch F, Laks J, et al. (2018) Can physical exercise modulate cortisol level in subjects with depression? A systematic review and meta-analysis. Trends Psychiatry Psychother 40: 360-368.

19. Stojan R, Voelcker-Rehage C (2019) A Systematic Review on the Cognitive Benefits and Neurophysiological Correlates of Exergaming in Healthy Older Adults. J Clin Med 8(5): E734.

$\begin{array}{ll}\text { BIOMEDICAL } & \text { Assets of Publishing with us } \\ \text { RESEARCHES } & \text { Global archiving of articles } \\ \text { - Immediate, unrestricted online access }\end{array}$

